\title{
Per aspera ad astra (Through hardship to the stars)
}

\author{
David M. Albala • Vladimir Mouraviev • \\ Srinivas Samavedi
}

Published online: 4 August 2013

(C) Springer-Verlag London 2013

"Every day you may make progress. Every step may be fruitful. Yet there will stretch out before you an everlengthening, ever-ascending, ever-improving path. You know you will never get to the end of the journey. But this, so far from discouraging, only adds to the joy and glory of the climb."

\section{- Sir Winston Churchill}

We are living in exciting times; robotics was thought to replace the mechanical actions of human activity. However, time has demonstrated that it cannot completely be done because only the central nervous system of the human brain can facilitate fine conscious movements at the highest level, especially those necessary to perform surgery. Therefore, a combination of the human brain and advanced robotic systems under the guidance of the former may lead us to tangible breakthroughs in surgery.

We like to make progress. But progress means nearing the goal. If along the journey we have taken a wrong turn, then going forward does not get you any nearer. If you are on the wrong road, progress requires making a U-turn to the right road. In this case, the man who turns back soonest is the most progressive man. In the late 1980s, the Army's Defense Advanced Research Projects Agency (DAPRA) investigated a remote, robotic triage system that could save

\section{M. Albala ( $\square)$}

Crouse Hospital, Syracuse, NY, USA

e-mail: dalbala@ampofny.com

V. Mouraviev

Associated Medical Professionals of NY, PLLC, Syracuse, NY, USA

\section{S. Samavedi}

Celebration Health, Florida, FL, USA soldiers' lives and keep Army doctors out of harm's way. A battlefield "surgical drone" never came to fruition, but the related idea of surgeons remotely manipulating robotic instruments with the help of a video feed led directly to the development of Intuitive Surgical's robotic system. Intuitive named its device after the renaissance painter Leonardo da Vinci, who first designed robots or "automations" as they were once called.

Approved by the Federal Drug Administration and installed in more than 1,900 hospitals and clinics, the Da Vinci robot-assisted laparoscopic surgery system is an excellent example of cutting-edge military technology converted to civilian use. Likewise, The Journal of Robotic Surgery is an excellent messenger of novel developments for a full army of robotic surgeons and scientists.

Thanks to the leadership of Dr. Vipul Patel as the previous Editor-in-Chief, the journal is now a strong vehicle to facilitate advances in robotics in surgery. Being a passionate innovator in the development of organ-sparing techniques for robotic-assisted laparoscopic prostatectomy for prostate cancer, he, by his own example, has been encouraging future generations of robotic surgeons in all surgical specialties. He has played a pivotal role in the development of robotic urological procedures as well as helping to advance robotics in other surgical disciplines. We thank Dr. Patel for his tremendous efforts and contributions in this process of promoting our journal to almost every clinic where robotics has been introduced into routine practice.

Our Editorial staff is ready to keep pace with the rapid advancements in robotic surgery. The Journal of Robotic Surgery aims to provide the reader with cutting-edge articles in all robotic surgical disciplines. We plan to further improve the quality of the scientific publications in order to have the journal indexed and increase the impact factor 
over the next 2-3 years. We plan to develop new sections including interviews with leading robotic surgeons and engineers, reviews of papers in robotic surgery published in other journals, and CME-bonus papers and case quizzes.

We are very excited to open a new chapter in the history of this journal and cordially invite the entire robotic community to actively participate in our activities on different levels, from submission of manuscripts to becoming a reviewer of papers. The robotic revolution in surgery began at the dawn of the new millennium and continues to see robust growth in all areas of surgery.

To find out more about the new Editorial team, you can read the Editor's biographies on the Journal homepage: http://www.springer.com/medicine/surgery/journal/11701. 